\title{
A COMPARATIVE STUDY OF CREATIVITY AND THE ROLE OF CHESS IN ITS DEVELOPMENT
}

\begin{abstract}
The article presents research data from 2004-2009. The creativity of younger schoolage children was examined. There has been a pattern here; the age of the child increases creativity decreases. The study was repeated in 2019. This time we got some interesting data. Creativity decreases at the beginning of junior school age, but increases at the end of age. I think this explanation should be explored, but the only change in the Armenian educational system is the introduction of the "Chess" subject. We believe that it has had a positive impact on the development of children's creativity.
\end{abstract}

Keywords: creativity, convergent thinking, divergent thinking, intelligence, giftedness, development,

\section{Introduction}

Before discussing the problem of creativity, let's understand what it means from the scientific point of view. The term "creativity" has been widely spread by J. Guilford's works (Guilford, 1950). He mentions two different thinking actions: convergent and divergent thinking. Convergent thinking operates in the case when a person who is solving a problem needs to find the only correct solution in different conditions. Although there may be some specific solutions, it is always limited. J. Guilford identified the convergencethinking ability of intellect tests, that is, the tests that measured the intelligence coefficient (IQ) (Guilford, 1959).

In a wider sense, creativity is regarded as an activity that is carried out in an uncertain situation and is aimed at achieving results where there is objective or subjective innovation. If we look at it from this point of view, creativity is not necessarily related to the types of activities that are traditionally creative, writing music, poems, painting and more. It is available in all cases when it is necessary to act in an uncertain situation when there are 
no ways to solve a problem and it is impossible to predict the changing conditions.

This problem has been further developed in research of Terman's work (Terman, 1925) on the characteristics and behavior of gifted students, there have been many debates and discussions centering around the notion of "giftedness." The most famous life-span study is Terman's which started in the 20s and is still ongoing (Terman, 1925). His 1528 subjects (672 girls and 856 boys) were selected on the basis of an IQ score of 135 or higher. His selection was based on IQ as the only crucial factor of giftedness. Actually, his selection was very careful and he did try to apply the high scientific standards of that time. In spite of the many deficits of such pioneering approach, his data provided us with rich material and new perspectives on the development of gifted subjects.

In 1926, Terman and K. Kock analyzed the biographies of 282 well-known people and based on their success, in the age between 17 and 26, tried to estimate the IQ. At the same time, they were based on Binets scale to evaluate intelligence at a childhood (Terman \& Oden, 1951). They found that children with intellectual giftedness were two years more advanced from their peers.

The intelligence coefficient was inspected in three stages: 1927-1928, 1932-1940, and in 1951-1952. The latest research was carried out by D. Feldman 60 years after the start of the research (Feldman 1979). He checked the successes of people chosen by Terman.

800 men, who were among those chosen and had 135 intellectual capacity, had 67 books (21 artistic and 46 monographs) printed at the age of 50, 78 of them were doctors of philosophy, 48 were doctors of medicine, and so on. 47 male names were included in the guide "1949 American Best Citizens". These figures were 30 times higher than the benchmarks of the comparative group. It should be noted that the children separated by $L$. Terman, were differed with early development (early, walk, speak, read, write, etc.). All intellectual children have successfully completed the school, most of them have received university education, and 200 people become Doctor of Science. Comparative research on the knowledge and skills of well-known people has shown that their intelligence coefficient is fairly high (Feldman, 1979).

However, as regards the results of creative success, they were not so definite. No one early intellectual chosen by Terman have ever been shown in any of the talented creators. None of them has made a significant contribution to the development of world culture (Feldman, 1979). 
Thus, an intellectually developed child is successfully adapted to society. Intellect is not an obstacle but a prerequisite for succeeding in a democratic society. But the high level of intelligence does not mean that this person will reach creative heights. However, there is also an opinion that there is some correlation between intelligence and creativity.

Terman, convinced by his own empirical data, came to the conclusion that intelligence is important, a necessary condition, but that a supportive home and school environment as well as high achievement motivation contribute in an essential way to the realization of high potential. More important creative abilities to create universal values (Feldman, 1979).

We conducted our research from 2004 to 2009. The purpose of the research was to identify gifted children, as the theme of my dissertation was "Causality of the Psychological Alienation in the Relationships of Gifted Children and Psychological Methods for Overcoming, so we had the task to identify gifted children, and the scientific criterion of giftedness was two: a high level of intellect and creativity. We have studied a large number of children aged 5-9 to choose from them gifted children. As a result of this research, we had a large number of research data related to creativity and intelligence. The study included 993 schoolchildren - 567 boys and 426 girls. The children were students of the A. Shirakatsi Lyceum- 472 children, from school number 87 - 209 children, and school number 31 - 312 children.

\section{Materials and methods}

For the study of creativity, we used the modified version of E. P. Torrance, "Differential Thinking" or Test of Creativity / Williams Modification, as well as various tasks have been selected by us, which were aimed to discover creativity. These tasks can have different solutions and original approach. 
Figure 1.

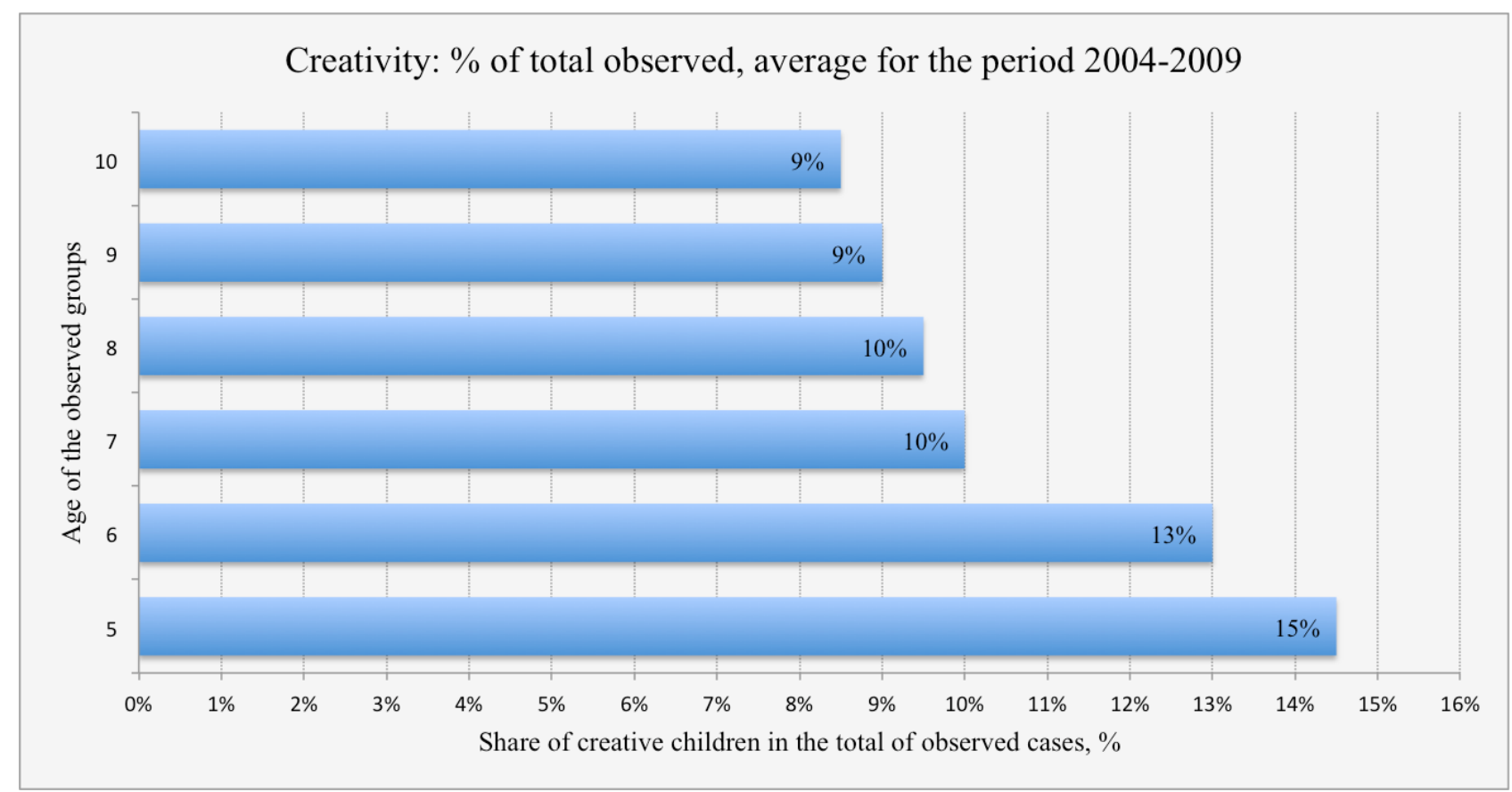

\section{Results and discussion}

During the analysis of the results of the creativity, we noticed regularity. As you can see from the graph, parallel to the age, the level of creativity decreases. This means that creativity in the group of younger children is higher than in older age groups. The reasons can be very different. However, we can conclude that the compulsory school programs Traditional approaches to teaching do not contribute to the development of such an important quality, such as creativity. We also think that nobody is skeptical of the idea that people with such capacities can develop science and technology, as well as through them humanity will overcome the challenges. In 2019, we have tried to re-explore the level of creativity, because changes have taken place in the educational system in Armenia: chess has become a mandatory subject in 2011, and our goal is to find out whether the introduction of this subject has any impact on creativity. The research has been organized and is being implemented in several schools in Yerevan - number 67, 2, 8, 150, 137, 129, as well as in some schools of Artashat and Echmiadzin. At this moment have been researched 201 schoolchildren - 109 boys and 92 girls.

To ensure the reliability of the research has been applied the same methods - E. P. Torrance's test of "Divergent thinking" or creativity and same tasks. Now we are going to present the results by percentage and graphic expression, as well as compare with previous results. 


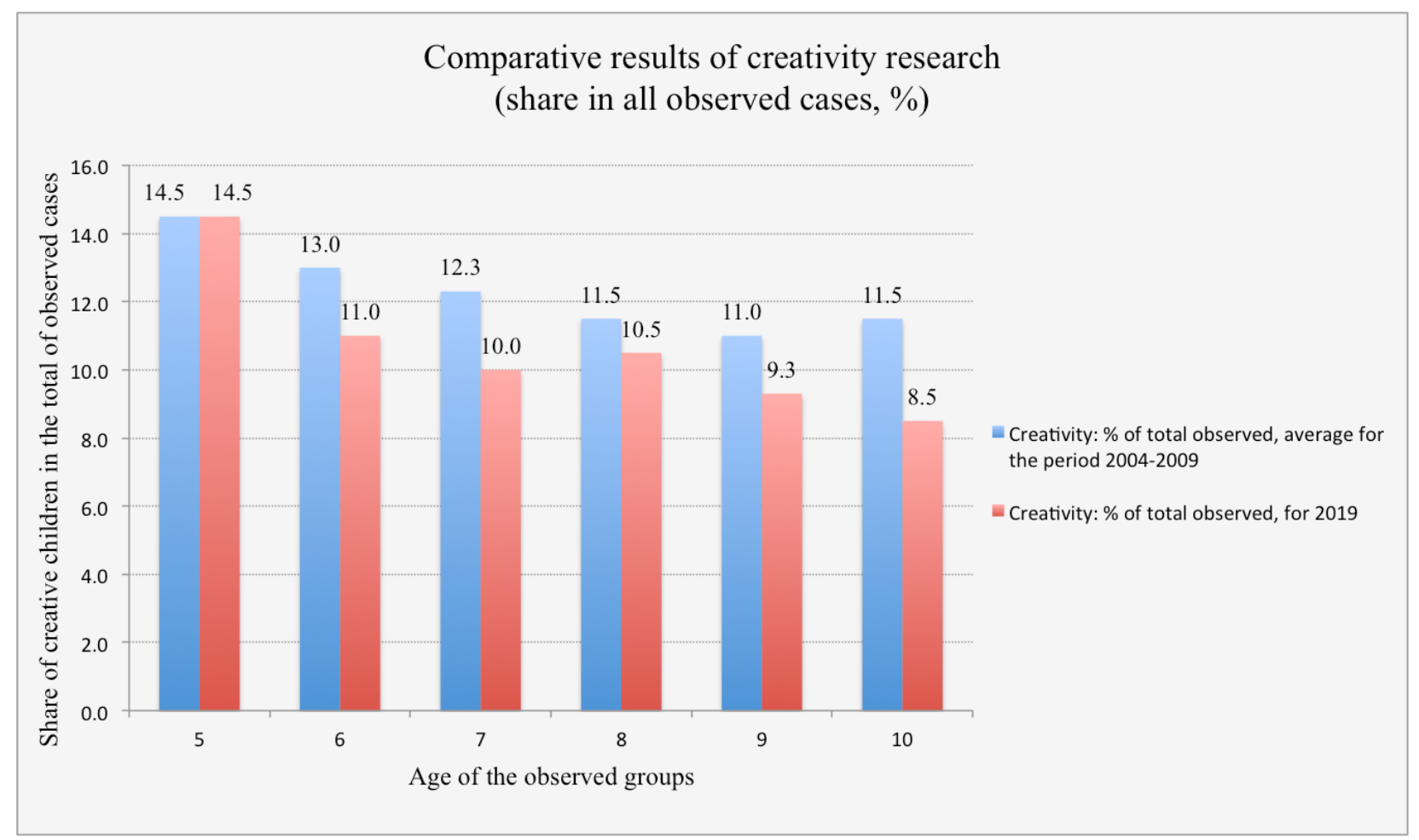

In the graph we can see, the data has changed. 6 and 7-year-olds about creativity level are reduced. We assume that the reason for these changes in the age to go to school. In other words, during the initial study they were going to school at the age of seven, and today at age 6 . As the child has a problem with adapting to a school situation, it affects creative thinking. But the continuation of the development is encouraging. The creativity gradually rises, though slightly. The downward trend is observed in the ages of 6 and 7 , but comparing with the previous years, we see that there is a tendency of growth.

\section{Conclusions}

Of course, one can argue and discuss reasons, what factors can have affected this change, but in the system of education in Armenia, crucial changes didn't happen and only the change is a chess subject's input as a compulsory.

We suppose that chess in education influences the development of creative thinking of children, which is very important.

\section{References}

Feldman, D., (1979). The Mysterious Case of Extreme Giftedness (p. 57). Chicago.

Guilford, J., (1950). Creativity. American Psychologist, (5), pp. 444-454. 
Guilford, J., (1959). Traits of Creativity, Creativity and its Cultivation (p. 3) New York.

Terman, L., (1925). Mental and Physical Traits of a Thousand Gifted Children. Genetic Studies of Genius (vol.1, p. 522) Stanford, CA.

Terman, L. M., \& Oden, M. H., (1951). The Stanford studies of the gifted (p. 20-45), NYC:

D. C., Heath. 